\title{
Capillary Electrophoresis Method for Determining the Content of Rutin in Oriental arborvitae
}

\author{
Xiaori Sun, Jinghui Zheng, Ting Zhou, Haixing Liua, \\ Chemistry \& Chemical and Environmental Engineering College, Weifang University, Weifang \\ 261061, P.R. China. \\ *, ahaixingliu@tom.com
}

\begin{abstract}
The experiment was carried out for the determination of rutin content in Oriental arborvitae by high performance capillary electrophoresis method. The borax solution as buffer solution was chosen, and its concentration was $20 \mathrm{mmol} / \mathrm{L}$ at a constant voltage of $20 \mathrm{kV}$ and injecting time of $10 \mathrm{~s}$. The detection wavelength was $254 \mathrm{~nm}$. Measured rutin content in Oriental arborvitae was 2.3491 $\mathrm{mg} / \mathrm{g}(\mathrm{RSD}=9.12 \%)(n=6)$. The recovery was in the range of $88 \%-118 \%(n=4)$.
\end{abstract}

Keywords: Capillary electrophoresis, Oriental arborvitae, Rutin.

\section{Introduction}

Oriental arborvitae is traditional Chinese medicine. It has the effect of cool blood and hemostasis, clear up the phlegm to stop coughing, hair growth and black hair. It is used for the treatment of vomiting blood, hemoptysis, hematochezia, cough with lung heat, blood fever, hair loss and premature graying hair [1]. It has the component of volatile oil, flavonoids, tannins, inorganic element, etc. It has the antibacterial, anti-inflammatory, hemostasis, preventing cough and dispelling phlegm, antitumor pharmacological action [2]. The inhibition effects of six kinds of plant extracts (including garlic,pomegranate,xanthium and so on) on pathogenic bacteria of some usual vegetable diseases were discussed by Meng [3].The results showed the inhibition rate of oriental arborvitae on the powdery mildew were better than that on other pathogenic bacteria.For screening the new botanical acaricide, the biological activities of 8 species of plant extracts were determined in laboratory using leaching stem immersion method by Jia et al. [4]. The results indicated that the biological activities of oriental arborvitae, Euphorbia australis L., Rumex japonicas Houtt and Punica granatum Linn. Were high. Luo et al [5] established a method for determining myricetin and quercetin in oriental arborvitae. The separation was carried out on an Inertsil-ODS-3(4.6mm $\times 250 \mathrm{~mm}, 5 \mu \mathrm{m})$ column, the mobile phase consisted of methanol- $0.01 \mathrm{~mol} / \mathrm{L}$ potassium dihydrogen phosphate solution - glacial acetic acid(37:63:1.5), with a flow rate of $1.0 \mathrm{~mL} / \mathrm{min}$ and the wavelength of $254 \mathrm{~nm}$. Cheng et al. [6] established an HPLC method for the determining isopimaric acid in the leaves of platycladus orientalis and its preparations cebai No. V capsula and isopimaric acid capsula to evaluate the quality of them. The Kromasil C18 ( $200 \mathrm{~mm} \times 4.6 \mathrm{~mm}, 5 \mu \mathrm{m}$ ) column was adopted. The mobile phase was composed of methyl alcohol - water - acetic acid (60:37:3) with flow rate of $1 \mathrm{~mL} / \mathrm{min}$ and wavelength of $231 \mathrm{~nm}$. Tan et al. [7] established an HPLC method for simultaneously determining the contents of myricetrin, quercitrin, yricetin, quercetin, kaempferol, amentoflavone and hinokiflavone in oriental arborvitae and oriental arborvitae Carbonisatum. The experiment were explored on Welch Ultimate LP-C18 column ( $4.6 \mathrm{~mm} \times 250 \mathrm{~mm}, 5 \mu \mathrm{m}$ ), mobile phase was methanol $0.2 \%$ phosphoric acid aqueous solution in a gradient elution mode and the wavelength was $330 \mathrm{~nm}$. Meng et al. [8] established an HPLC method for the simultaneous determining myricitrin, quercitrin, amentoflavon and hinokiflavon in order to provide the basis for the quality control and exploitation of platycladi cacumen. A Waters ODS C18 column ( $4.6 \mathrm{~mm} \times 150 \mathrm{~mm}, 5 \mu \mathrm{m})$ was applied with a gradient elution of methanol-water containing $0.5 \%$ acetic acid and the flow rate of $0.8 \mathrm{~mL} / \mathrm{min}$ and the detection wavelength of $340 \mathrm{~nm}$. Shan et al [9] investigated the flavonoids in oriental arborvitae. The constituents in oriental arborvitae were determined by UPLC-MS. A Waters BEH C18 column $(2.1 \mathrm{~mm} \times 150 \mathrm{~mm}, 1.7 \mu \mathrm{m})$ was adopted with a gradient elution of methanol water containing $0.2 \%$ formic acid. The mass spectrometer equipped with electrospay ionization source was used as defector and operated in data was collected under the negative ion modes. Eleven constituents were identified. 
$\mathrm{Gu}$ et al [10] investigated the inhibition of pancreatic lipase and antioxidation of different extracts from oriental arborvitae leaves in vitro. The inhibitory activities of pancreatic lipase of ethanol extract, petroleum aether extract, ethyl acetate extract, n-butyl alcohol extract and water extract were assayed in vitro. The antioxidant activities of these extracts were assessed by two complementary test systems, namely 1,1-diphenyl-2-picrylhydrazyl (DPPH) radical scavenging activity and ferric reducing antioxidant power (FRAP) assay. The ethyl acetate extract indicated strong activity in the inhibitory activities of pancreatic lipase. Li et al [11] extracted polysaccharide from oriental arborvitae leaf and analyze its antioxidant activity. With distilled water as the extraction agent, using the method of ultrasonic extraction of Oriental arborvitae polysaccharide, the main factors affecting the extraction yield of polysaccharides by single factor and orthogonal experiments, and the best extraction technology conditions were obtained. The antioxidant activity of polysaccharides from oriental arborvitae leaf was studied by superoxide anion free radical scavenging assays. The optimum ultrasonic extraction conditions were solid - liquid ratio $(\mathrm{g}: \mathrm{mL}) 1: 35$, temperature $60^{\circ} \mathrm{C}$, ultrasonic power $180 \mathrm{~W}$ and ultrasonic action time $40 \mathrm{~min}$, under this condition, the oriental arborvitae leaf polysaccharide's extraction quantity was $8.35 \%$. Ding et al [12] established a new determining method for quercitrin from oriental arborvitae leaves by capillary-zone electrophoresis (CZE). The optimized CZE conditions was the buffer composed of $30 \mathrm{mmol} / \mathrm{L}$ borax-boric acid (volume 1:3) with the separation voltage of $25 \mathrm{kV}$ and the wavelength of $254 \mathrm{~nm}$.

\section{Experimental Section}

\subsection{Instruments and Reagents}

Experimental instruments: CL-1030-type high performance capillary electrophoresis (Beijing Cailu Scientific Instrument Co., Ltd.); HW2000-type chromatography workstation (Nanjing Qianpu Software Ltd.); Capillary (75 $\mu \mathrm{m}$ inner diameter, $52 \mathrm{~cm}$ overall length, $44 \mathrm{~cm}$ effective length) from Hebei Yongnian Ruifeng Chromatographic Devices Co., Ltd.). Rutin ( Shanghai Alading Biochemical Technology Co., Ltd.); Oriental arborvitae (Weifang pharmaceutical Co., Ltd.); Other reagents used in the experiments were all analytical grade; Double-distilled water was used.

\subsection{Experimental Methods}

Before the start of the experiment, capillary was successively washed with $1 \mathrm{~mol} \cdot \mathrm{L}^{-1}$ hydrochloric acid solution, double-distilled water, $1 \mathrm{~mol} \cdot \mathrm{L}^{-1}$ sodium hydroxide solution, double-distilled water, buffer solution, each for $5 \mathrm{~min}$. After three times running, capillary was cleaned again using the above method.

Measurements were carded out at $16 \mathrm{kV}$ voltage and experimental temperature at $20^{\circ} \mathrm{C}$. UV detection wavelength was $254 \mathrm{~nm}$. Injection time was $10 \mathrm{~s}$ ( $7.5 \mathrm{~cm}$ height difference).

\subsection{Sample Preparation}

Oriental arborvitae sample solution: Oriental arborvitae powder was accurately weighed $1.4212 \mathrm{~g}$, added $40 \mathrm{~mL}$ water, extracted time of $24 \mathrm{~h}$ at $20^{\circ} \mathrm{C}$, filtered, washed and set the volume to $50 \mathrm{~mL}$ that was the Oriental arborvitae sample solution.

Rutin standard solution: Rutin was accurately weighed $50 \mathrm{mg}$, added $50 \mathrm{~mL}$ water with $30 \%$ methanol.

\section{Results and Discussion}

\subsection{Selection Electrophoresis Conditions}

Based on past experiment experience, we chose $20 \mathrm{mmol} / \mathrm{L}$ borax solution as a running buffer solution.

According to the literature, Rutin absorption wavelength was at $254 \mathrm{~nm}$, so we chose the $254 \mathrm{~nm}$ detection wavelength. 


\subsection{Quantitative Analysis}

\subsubsection{Standard Curve}

First, Rutin standard solution that the concentration were $0.2,0.1,0.05,0.025,0.0125,0.00625$ $\mathrm{mg} / \mathrm{mL}$ was prepared. Each standard solution was run for three times under the above electrophoresis conditions and the results averaged. Taking concentration as the abscissa and peak area as the ordinate, the standard curve was drew. Linear regression equation of Rutin (peak area: $y \mu V \cdot s$, density: $x$ $\mathrm{mg} / \mathrm{mL})$ and the linear range was as follows: $\mathrm{Y}=3137.75+331536.12 \mathrm{X}(\mathrm{r}=0.973), 0.00625 \sim 0.2$ $\mathrm{mg} / \mathrm{mL}$.

\subsubsection{Precision Test}

A Rutin standard solution precisely drew and continuously injected for sixt times under electrophoretic separation conditions, the RSD of Rutin peak area were $8.7 \%$.

\subsubsection{Determination of Sample Content}

Under selected electrophoresis conditions, Oriental arborvitae sample solution was run. Separation chromatogram of the Oriental arborvitae sample solution was showed in Figure 1. Measured Rutin content in Oriental arborvitae was $2.3491 \mathrm{mg} / \mathrm{g}(\mathrm{RSD}=9.12 \%)(\mathrm{n}=6)$.

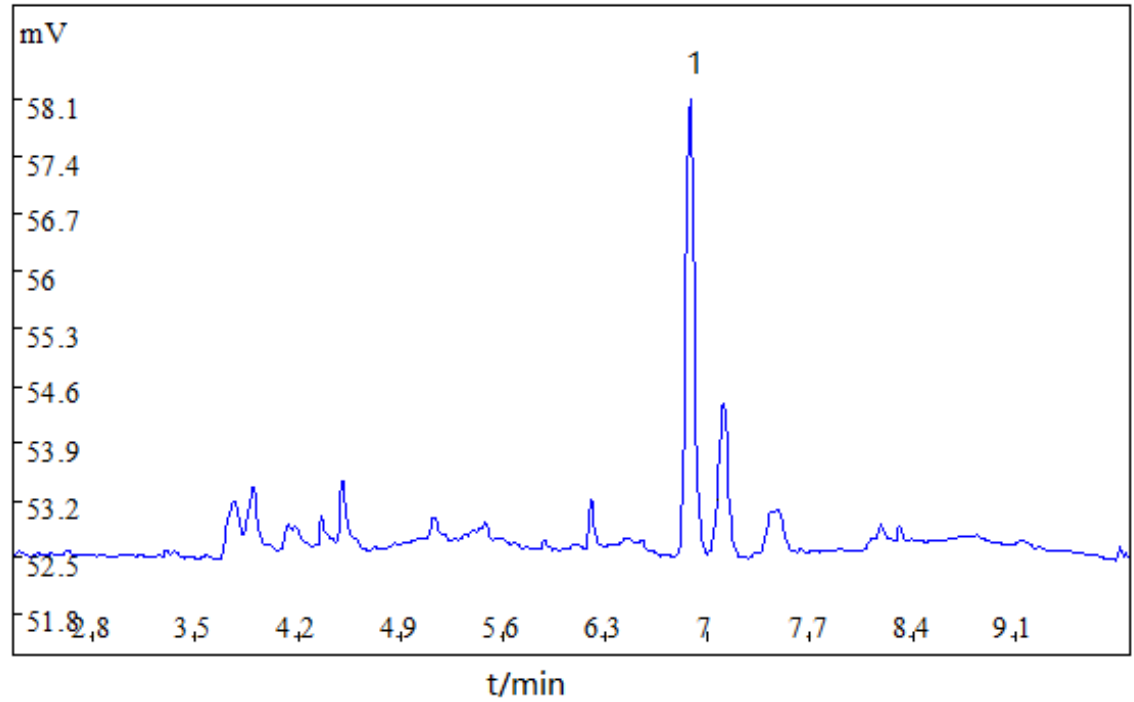

Fig. 1 Electrophorogram of Oriental arborvitae sample solution 1- Rutin

\subsubsection{Recovery}

After determination for six times, the recovery of Rutin content in Oriental arborvitae sample was in the range of $88 \%-118 \%(n=4)$.

\section{Acknowledgments}

This study were supported by the Natural Science Foundation of Shandong Province (No. ZR2010BL025), Open Project of State Key Laboratory of Supramolecular Structure and Materials (No. sklssm201323)(Jilin University), State Key Laboratory of Inorganic Synthesis and Preparative Chemistry (No. 2011-13)(Jilin University).

\section{References}

[1]. Chinese pharmacopoeia commission, Pharmacopoeia of the People's Republic of China(A), Beijing, China Medical Science and Technology Press, 2010: 200. 
[2]. Yan Huang, Cheng-zhi Liang, Yi Zhang, Pharmacological study on the oriental arborvitae and its application in Dermatology, Yunnan journal of traditional Chinese medicine, 2007, 28 (3): $42-44$.

[3]. Zhao-ming Meng, Study on Antibacterial Activities of Six kinds of Plant Extracts, Journal of Anhui Agri. Sci. 2011, 39( 8) : 4570-4571.

[4]. Fuli Jia, Yijuan Chen, Jia Chen, et al, Biologica 1Activity of Extracts from 8 Species of Plants Against tetranychus Cinnabarius, ChineseAgricultural Science Bulletin, 2011, 27(24): 286-291.

[5]. Shi-heng Luo, Fu-rong Li, Xi-fang Wang, et al, HPLC determination of myricetin and quercetin in Platycladus orientalis ( L ) Franco from different collection periods and different areas, Chin J Pharm Anal, 2013, 33( 3): 399-403.

[6]. Li-fang Cheng, Xiu-jun Cui, Hai-yong Ma, Determination of isopimaric acid in leaves of Platycladus orientalis and its preparations Cebai No. V Capsula and Isopimaric Acid Capsula by HPLC, Chinese Traditional and Herbal Drugs, 2007, 38(4): 521-523.

[7]. Xiao-liang Tan, Rui-hai Li, Simultaneous determination of seven constituents in Cacumen Platycladi and Cacumen Platycladi Carbonisatum, Chinese Traditional Patent Medicine, 2015, 37(12): 2715-2718.

[8]. Li-juan Meng, Song Cheng, Ying-ni Pan, et al, Simultaneous determination of the contents of four flavonoids in Platycladi Cacumen by HPLC, Journal of Shenyang Pharmaceutical University, 2014, 31(2):107-111.

[9]. Ming-qiu Shan, Wen Qian, Jing Gao, et al, Analysis of flavonoids in Platycladi Cacumen by UPLC-MS, China journal of Chinese materia medica, 2011, 36(12): 1626-1629.

[10]. Jun Gu, Yi Sun, Study on Inhibition of Pancreatic Lipase and Antioxidation from Platycladus orientalis Leaves in vitro, Chinese Journal of Experimental Traditional Medical Formulae, 2015, 21(14): 141-144.

[11]. Yuan-hui Li, Quan-tai Wen, Zhao-ping Zhang, et al, Extraction Process of Polysaccharides from Cebaiye ( Platycladus Orientalis Leaf) and Study on Its Antioxidant Activity, Chinese medicine science and technology, 2016, 23(1): 40-42.

[12]. Hong-mei Ding, Zhen-hua Sheng, Er-ning Ge, Determination of Quercitrin from Platycladus orientalis Leaves by Capillary-zone Electrophoresis, Chinese Journal of Experimental Traditional Medical Formulae, 2010, 16(11): 73-75. 